\title{
Quantify entanglement by concurrence hierarchy
}

\author{
Heng Fan, Keiji Matsumoto, Hiroshi Imai \\ Quantum computing and information project, ERATO, \\ Japan Science and Technology Corporation, \\ Daini Hongo White Bldg.201, Hongo 5-28-3, Bunkyo-ku, Tokyo 133-0033, Japan.
}

\begin{abstract}
We define the concurrence hierarchy as $d-1$ independent invariants under local unitary transformations in d-level quantum system. The first one is the original concurrence defined by Wootters et al 1.21 in 2-level quantum system and generalized to d-level pure quantum states case. We propose to use this concurrence hierarchy as measurement of entanglement. This measurement does not increase under local quantum operations and classical communication.
\end{abstract}

\section{INTRODUCTION}

Entanglement plays a central role in quantum computation and quantum information [3]. One of the main goals of theory of entanglement is to develope measures of entanglement. Several measures of entanglement are proposed and studied according to different aims, including entanglement of formation, entanglement of distillation, entanglement cost etc. [4, 5].

Perhaps one of the most widely accepted measures of entanglement is entanglement of formation $E_{f}$ which provides a very good measurement of entanglement asymptotically. For a pure bipartite quantum state $\rho=|\Phi\rangle\langle\Phi|$ shared by $\mathrm{A}$ and $\mathrm{B}$, entanglement of formation is defeined by von Neumann entropy of reduced density matrix $E_{f}(\rho)=$ $-\operatorname{Tr} \rho_{A} \log \rho_{A}$, where $\rho_{A}=\operatorname{Tr}_{B} \rho$. For mixed state, the entanglement of formation takes the form

$$
E_{f}(\rho)=\inf \sum_{j} p_{j} E_{f}\left(\Phi_{j}\right)
$$

where the infimum is taken over all pure-state decompositions of $\rho=\sum_{j} p_{j}\left|\Phi_{j}\right\rangle\left\langle\Phi_{j}\right|$. For mixed state, this definition is operational difficult because it requires finding the minimum average entanglement over all possible pure-state decompositions of the given mixed state. In d-dimension, the explicit expression of entanglement of formation is only found for several special types of mixed state, for example, the isotropic states [6] and Werner states [7]. However, the explicit formulas have been found for the 2-level quantum system by Wootters et al [1,2]. Here we briefly introduce the results by Wootters et al. The entanglement of formation of an arbitrary state $\rho$ is related to a quantity called concurrence $C(\rho)$ by a function

$$
E_{f}(\rho)=\epsilon(C(\rho))=h\left(\frac{1+\sqrt{1-C^{2}(\rho)}}{2}\right),
$$

where $h(x)=-x \log x-(1-x) \log (1-x)$ is the binary entropy function. The entanglement of formation is monotonically increasing with respect to the increasing concurrence. The concurrence is defined by an almost magic formula,

$$
C(\rho)=\max \left\{0, \lambda_{1}-\lambda_{2}-\lambda_{3}-\lambda_{4}\right\}
$$

where the $\lambda_{i}$ 's are the square root of the eigenvalues of $\rho \tilde{\rho}$ in descending order. And $\tilde{\rho}=\left(\sigma_{y} \otimes \sigma_{y}\right) \rho^{*}\left(\sigma_{y} \otimes \sigma_{y}\right)$, where $\sigma_{y}$ is the Pauli matrix. For pure state $|\Phi\rangle=\alpha_{00}|00\rangle+\alpha_{01}|01\rangle+\alpha_{10}|10\rangle+\alpha_{11}|11\rangle$, the concurrence takes the form

$$
C(\Phi)=\left|\left\langle\Phi\left|\sigma_{y} \otimes \sigma_{y}\right| \Phi^{*}\right\rangle\right|=2\left|\alpha_{00} \alpha_{11}-\alpha_{01} \alpha_{10}\right|
$$

Because of the relation between concurrence and entanglement of formation, we can use directly the concurrence as the measure of entanglemnet.

One important aim in formulating the measures of entanglement is to find whether a bipartite state is separable or not because the entanglement state has some useful applications, for example, teleportation 《8] quantum cryptography by using EPR pairs [9]. In 2-level quantum system, the Peres-Horodeckis [10,11] criterion is a convenient method. And the concurrence provide another method. If the concurrence is zero, the quantum state is separable, otherwise it is entangled. For general mixed state in d-dimension, we need yet to find an operational method to distinguish separability and entanglement. 
For pure state in d-dimension, the measure of entanglement is largely solved by entanglement of formation. We can use it to distinguish whether a pure state is separable or not and to find the amount of entanglement. However, to complete characterizing the entanglement, one quantity seems not enough. A simple example is [12]:

$$
\begin{aligned}
& |\psi\rangle=1 / \sqrt{2}(|00\rangle+|11\rangle), \\
& |\phi\rangle=\sqrt{x} / \sqrt{2}(|00\rangle+|11\rangle)+\sqrt{1-x}|22\rangle
\end{aligned}
$$

When $x \approx 0.2271$ is a root of equation $x^{x}[2(1-x)]^{1-x}=1$, the entanglement of formation equal to 1 for both $|\psi\rangle$ and $|\phi\rangle$. However, they can not be transformed to each other by local operations and classical communication (LOCC).

Because concurrence provide a measure of entanglement in 2-level system, it is worth generalizing concurrence to higher dimension. There are several proposals for the case of pure states [13, 14, 15, 12, 16, 17, . Uhlmann generalized the concurrence by considering arbitrary conjugations acting on arbitrary Hilbert spaces $13 \|$. Rungta et al generalized the spin flip operator $\sigma_{y}$ to a universal inverter $S_{d}$ defined as $S_{d}(\rho)=1-\rho$, so the pure state concurrence in any dimension takes the form

$$
\begin{aligned}
C^{\prime}(\Phi) & =\sqrt{\left\langle\Phi\left|S_{d_{1}} \otimes S_{d_{2}}(|\Phi\rangle\langle\Phi|)\right| \Phi\right\rangle} \\
& =\sqrt{2\left[1-\operatorname{Tr}\left(\rho_{A}^{2}\right)\right]} .
\end{aligned}
$$

There is a simple relation between these two generalizations pointed out by Wootters [18]. Another generalization proposed by Albeverio and Fei [15] by using an invariants under local unitary transformations turns out to be the same as that of Rungta et al up to a whole factor. They define the concurrence as

$$
C(\Phi)=\sqrt{\frac{d}{d-1}\left[1-\operatorname{Tr}\left(\rho_{A}^{2}\right)\right]} .
$$

Let's analyze the example (5) again by the generalized concurrence. When $x=1 / 3$ is a root of equation $(3 x-1)(x-1)=$ 0 , the concurrence of $|\psi\rangle$ and $|\phi\rangle$ are equal. But still $|\psi\rangle$ and $|\phi\rangle$ can not be transformed to each other by LOCC.

As already noticed and conjectured by many researchers, one quantity perhaps is not enough to measure all aspects of entanglement [19, 20,21,22, 23, 24, 25, 26], see [27] for a review, and the geometric properties of entanglement was investigated in [28. As the question of separability, Peres-Horodeckis 10,11] criterion is enough for bipartite 2-level quantum system. For higher dimension, if we want to find whether a bipartite state is entangled, besides partial transposition operation proposed by Peres [10], we need to find other positive but not completely positive maps. Presently, how to find whether a bipartite state in $C^{d_{1}} \times C^{d_{2}}$ is entangled is still an open problem.

\section{DEFINITION OF CONCURRENCE HIERARCHY}

In this paper, we propose to use the concurrence hierarchy to quantify the entanglement for d-dimension. We restrict ourself to $C^{d} \otimes C^{d}$ bipartite pure state. A general bipartite pure state in $C^{d} \otimes C^{d}$ can be written as

$$
|\Phi\rangle=\sum_{i, j=0}^{d-1} \alpha_{i j}|i j\rangle
$$

with normalization $\sum_{i j} \alpha_{i j} \alpha_{i j}^{*}=1$. We define a matrix $\Lambda$ with entries $\Lambda_{i j}=\alpha_{i j}$. The reduced density matrix can be denoted as $\rho_{A}=\operatorname{Tr}_{B} \rho=\Lambda \Lambda^{\dagger}$. Under a local unitary transformation $U \otimes V$, the matrix $\Lambda$ is changed to $\Lambda \rightarrow U^{t} \Lambda V$, where the superindex $t$ represents transposition. And the redeced density operator thus is transformed to

$$
\rho_{A} \rightarrow\left(U^{t} \Lambda V\right)\left(V^{\dagger} \Lambda^{\dagger} U^{t \dagger}\right)=U^{t} \Lambda \Lambda^{\dagger} U^{t \dagger}
$$

In 2-dimension, it was point out by Linden and Popescu [29], there is one no-trivial invariant under local unitary transformations $I=\operatorname{Tr}\left(\Lambda \Lambda^{\dagger}\right)^{2}$. In general d-dimension, it was pointed out by Albeverio and Fei that there are $d-1$ independent invariants under local unitary transformations $I_{k}=\operatorname{Tr}\left(\Lambda \Lambda^{\dagger}\right)^{k+1}$. When $k=0$, it is just the normalization equation $I_{0}=\sum_{i j} \alpha_{i j} \alpha_{i j}^{*}=1$. For $k=1, \cdots, d-1, I_{k}$ are $d-1$ independent invariants under local unitary transformations. Then they generalize the concurrence as the formula (7) and one relation be calculated as

$$
1-\operatorname{Tr} \rho_{A}^{2}=I_{0}-I_{1}=\frac{1}{2} \sum_{i, j, k, m}^{d}\left|\alpha_{i k} \alpha_{j m}-\alpha_{i m} \alpha_{j k}\right|^{2}
$$


When $C(\Phi)=0$, it is separable; when $C(\Phi) \neq 0$, it is entangled; when $C(\Phi)=1$, it is maximally entangled state. For a pure state $|\Phi\rangle$ as in (8), when $\alpha_{i k} \alpha_{j m}=\alpha_{i m} \alpha_{j k}$ for all $i, j, k, m$, it can be written as a product form and thus separable. It is a rather intuitive idea to use quantity (10) as the measure of entanglement. And all proposals of generalization of concurrence actually lead to this result. And also when $C(\Phi) \neq 0$, state $|\Phi\rangle$ is entangled. However, our opinion is that this quantity is necessary but not enough. In quantifying the entanglement, the entanglement is dealed independently by restricted to every 2-level system. For example, suppose $\left|\Phi^{\prime}\right\rangle$ takes the form

$$
\left|\Phi^{\prime}\right\rangle=\alpha_{00}|00\rangle+\alpha_{11}|11\rangle+\alpha_{22}|22\rangle .
$$

Actually we can always change a pure state $|\Phi\rangle$ to this form by Schmidt decomposition. The states $\alpha_{00}|00\rangle+\alpha_{11}|11\rangle$, $\alpha_{00}|00\rangle+\alpha_{22}|22\rangle$ and $\alpha_{11}|11\rangle+\alpha_{22}|22\rangle$ are considered independently in (10) and the entanglement in every 2-level system is sumed together $C(\Phi)=\left|\alpha_{00} \alpha_{11}\right|^{2}+\left|\alpha_{00} \alpha_{22}\right|^{2}+\left|\alpha_{11} \alpha_{22}\right|^{2}$. As already pointed out previously, when $x=1 / 3$, the concurrence of $|\psi\rangle$ and $|\phi\rangle$ in (5) are equal but they can not be transformed to each other by LOCC. Our idea here is that besides the concurrence in the form (10), we should also quantify it by other quantities. For example, the state $\left|\Phi^{\prime}\right\rangle$ in (11), we can quantify the entanglement by

$$
C_{3}\left(\Phi^{\prime}\right)=\left|\alpha_{00} \alpha_{11} \alpha_{22}\right|^{2},
$$

up to a normalized factor. In this quantity we just consider the entanglement in all 3 levels. Apparently, $C_{3}\left(\Phi^{\prime}\right)=0$ does not mean the state $\left|\Phi^{\prime}\right\rangle$ is separable. So both this quantity and (10) are necessary in quantifying the entanglement in 3-level quantum system. We call these two quantities as concurrence hierarchy for 3-level system. The example (5) thus can be distinguished as follows. If you let both $C(\psi)=C(\phi)$ and $C_{3}(\psi)=C_{3}(\phi)$, we can find just one solution $x=1$, i.e. $|\psi\rangle=|\phi\rangle$. In case $x=1 / 3$, though the two level concurrences defined in (7) for $|\psi\rangle$ and $|\phi\rangle$ are equal, their 3-level concurrences are different, $C_{3}(\psi)=0$ while $C_{3}(\phi)=1 / 54$. The structure of their concurrence hierarchy is different. So, they can not be transformed to each other by LOCC.

Next, we give our precise definition of concurrence hierarchy. Suppose a bipartite pure state (8) shared by A and B, $\lambda_{\Phi}=\left\{\lambda_{0}^{\downarrow}, \cdots, \lambda_{d-1}^{\downarrow}\right\}$ denotes the vector of eigenvalues of the reduced density operator $\rho_{A}=\operatorname{Tr}_{B}(|\Phi\rangle\langle\Phi|)$ in decreasing order. In other words $\lambda_{j}^{\downarrow}, j=0, \cdots, d-1$ are square of singular values of matrix $\Lambda$.

Definition: The concurrence hierarchy of the state $|\Phi\rangle$ is defined as

$$
C_{k}(\Phi)=\sum_{\substack{0 \leq i_{0}<i_{1}<\cdots<i_{k} \leq(d-1) \\ k=1,2, \cdots, d-1 .}} \lambda_{i_{0}}^{\downarrow} \lambda_{i_{1}}^{\downarrow} \cdots \lambda_{i_{k}}^{\downarrow},
$$

We propose to use this concurrence hierarchy to quantify the entanglement of the state $|\Phi\rangle$.

The first level concurrence is trivial since it is just the normalization condition $C_{1}(\Phi)=\sum_{i=0}^{d-1} \lambda_{i}^{\downarrow}=1$. The two level concurrence is the d-dimension generalization of concurrence proposed by Rungta et al [14] and Albeverio et al [15] up to a whole factor. In 2-dimension, there are just one non-trivial concurrence which is the original concurrence proposed by Wootters et al [1,2]. In d-dimension, the concurrence hierarchy consists of $d-1$ independent non-trivial concurrences. The result of 3 level concurrence in 3-dimension is already presented in (12). This concurrence hierarchy is invariant under local unitary transformations and can be represented in terms of invariants $I_{k}=\operatorname{Tr}\left(\Lambda \Lambda^{\dagger}\right)^{k+1}$ [15]. It should be noted that a similar idea as this paper was also proposed by Sinolecka, Zyczkowski and Kus [26]. We give an example to show one relation for 3 level concurrence of state $|\Phi\rangle$ in (8),

$$
\begin{aligned}
C_{3}(\Phi)= & \sum_{0 \leq i_{0}<i_{1}<i_{2} \leq(d-1)} \lambda_{i_{0}}^{\downarrow} \lambda_{i_{1}}^{\downarrow} \lambda_{i_{2}}^{\downarrow} \\
= & 1+2 I_{2}-3 I_{1} \\
= & \frac{1}{6} \sum_{i j k l m r} \mid \alpha_{i j} \alpha_{k l} \alpha_{m r}+\alpha_{k j} \alpha_{m l} \alpha_{i r}+\alpha_{m j} \alpha_{i l} \alpha_{k r} \\
& -\alpha_{m j} \alpha_{k l} \alpha_{i r}-\alpha_{i l} \alpha_{k j} \alpha_{m r}-\left.\alpha_{i j} \alpha_{m l} \alpha_{k r}\right|^{2},
\end{aligned}
$$

where terms inside $|\cdot|$ correspond to determinants of the $3 \times 3$ submatrix of $\Lambda$ with row indices $i, k, m$ and column indices $j, l, r$. When $|\Phi\rangle$ is separable, all concurrences in the hierarchy are zeros except the trivial one. If the Schmidt number (rank) of $\rho_{A}$ for state $|\Phi\rangle$ in (8) is $k, 1 \leq k \leq d$, all higher level concurrences $C_{j}(\Phi)=0, j>k$. This is simple because all eigenvalues of $\rho_{A}$ are non-negative. 


\section{A SIMPLE METHOD TO CALCULATE THE CONCURRENCE HIERARCHY AND ENTANGLEMENT CAN BE QUANTIFIED BY CONCURRENCE HIERARCHY}

The concurrence hierarchy can be calculated by its definition (13). The 2,3-level concurrences can be calculated directly by relations (10,14). Here we show all concurrences in the hierarchy can be calculated similarly. According to some results in linear algebra, see for example Ref. [31], the concurrence hierarchy $C_{k}(\Phi)$ equal to the sums of the $k$-by- $k$ principal minors of reduced density operator $\Lambda \Lambda^{\dagger}$. And it is known that these quantities are invariant under unitary transformations $U \Lambda \Lambda^{\dagger} U^{\dagger}$. This leads straightforward to the result that for a bipartite pure state (8), the concurrence hierarchy $C_{k}(\Phi)$ are invariant under local unitary transformations. For convenience, we adopt the same notations as that of Ref. [31]. Let $\beta, \gamma \subseteq\{0, \ldots, d-1\}$ be index sets, each of cardinality $k, k=1, \cdots, d$. According to Cauchy-Binet formula, we have the following relations:

$$
\begin{aligned}
C_{k}(\Phi) & =\sum_{\beta} \operatorname{det} \rho_{A}(\beta, \beta) \\
& =\sum_{\beta} \sum_{\gamma} \operatorname{det} \Lambda(\beta, \gamma) \operatorname{det} \Lambda^{\dagger}(\gamma, \beta) \\
& =\sum_{\beta} \sum_{\gamma}|\operatorname{det} \Lambda(\beta, \gamma)|^{2},
\end{aligned}
$$

where we use the relation $\rho_{A}=\Lambda \Lambda^{\dagger}$, and the notation $\operatorname{det} \Lambda(\beta, \gamma)$ means the determinant of submatrix $\Lambda$ with row and column index sets $\beta$ and $\gamma$. When the cardinality $k=2,3$, we recover the previous results (10,14). So, we do not need to calculate the eigenvalues of the reduced density operator to find the concurrence hierarchy, we can calculate the concurrence hierarchy directly by summing the determinants of all $k$-by- $k$ submatrices of $\Lambda$.

Next, we show the concurrence hierarchy cannot increase under LOCC. We use the theorem proposed by Nielsen by majorization scheme [30]. For convenience, we use the same notations as that of Ref. 31] and Nielsen. The elements of vectors $x=\left\{x_{0}^{\downarrow}, \cdots, x_{d-1}^{\downarrow}\right\}$ and $y=\left\{y_{0}^{\downarrow}, \cdots, y_{d-1}^{\downarrow}\right\}$ are ordered in decreasing order. We say that $x$ is majorized by $y, x \prec y$, if $\sum_{j=0}^{k} x_{j}^{\downarrow} \leq \sum_{j=0}^{k} y_{j}^{\downarrow}, k=0, \cdots, d-1$ and the equality holds when $k=d-1$.

Theorem 1 by Nielsen [30]: $|\Psi\rangle$ transforms to $|\Phi\rangle$ using LOCC if and only if $\lambda_{\Psi}$ is majorized by $\lambda_{\Phi}$,

$$
|\Psi\rangle \rightarrow|\Phi\rangle \quad \text { iff } \quad \lambda_{\Psi} \prec \lambda_{\Phi}
$$

Now we propose our theorem by directly using Nielsen theorem.

Theorem 2: $|\Psi\rangle$ transforms to $|\Phi\rangle$ using LOCC, the concurrence hierarchy of $|\Psi\rangle$ is no less than that of $|\Phi\rangle$. And explicitly, if $|\Psi\rangle \rightarrow|\Phi\rangle$, then $C_{k}(\Psi) \geq C_{k}(\Phi), k=1, \cdots, d$.

The proof of this theorem is as follows. Because of Nielsen theorem, $|\Psi\rangle \rightarrow|\Phi\rangle$ then we have $\lambda_{\Psi} \prec \lambda_{\Phi}$. Because $-C_{k}, k=1, \cdots, d$ are isotone functions [31], i.e. if $\lambda_{\Psi} \prec \lambda_{\Phi}$ then $-C_{k}(\Psi) \leq-C_{k}(\Phi)$. Thus we have $C_{k}(\Psi) \geq$ $C_{k}(\Phi), k=1, \cdots, d$. Here we mainly use the fact that each $C_{k}$ are Schur-concave functions, see [31.

It is well known that minus entropy function is isotone, so the entanglement of formation cannot increase under LOCC. Here we show the concurrence hirerarchy cannot increase under LOCC.

\section{APPLICATIONS OF CONCURRENCE HIERARCHY}

According to the theorem 2 , if some of the relations $C_{k}(\Psi) \geq C_{k}(\Phi), k=1, \cdots, d$ do not hold, $|\Psi\rangle$ and $|\Phi\rangle$ can not be transformed to each other by LOCC. Here we analyze an example raised by Nielsen [30],

$$
\begin{aligned}
|\Psi\rangle & =\sqrt{0.5}|00\rangle+\sqrt{0.4}|11\rangle+\sqrt{0.1}|22\rangle, \\
|\Phi\rangle & =\sqrt{0.6}|00\rangle+\sqrt{0.2}|11\rangle+\sqrt{0.2}|22\rangle .
\end{aligned}
$$

According to Nielsen theorem, neither $|\Psi\rangle \rightarrow|\Phi\rangle$ nor $|\Phi\rangle \rightarrow|\Psi\rangle$. Here we analyze this example by calculating their concurrence hierarchy. We can find

$$
\begin{array}{r}
C_{2}(\Psi)=0.29>C_{2}(\Phi)=0.28 \\
C_{3}(\Psi)=0.020<C_{3}(\Phi)=0.024
\end{array}
$$


It follows from theorem 2 that neither $|\Psi\rangle \rightarrow|\Phi\rangle$ nor $|\Phi\rangle \rightarrow|\Psi\rangle$. We can roughly interprete the reason as that the 2-level entanglement of $|\Psi\rangle$ is larger than that of $|\Phi\rangle(18)$, but the 3-level entanglement of $|\Psi\rangle$ is less than that of $|\Phi\rangle$ (19). So we cannot transform them to each other by LOCC.

It should be noted that the inverse of theorem 2 is not ture. That means even we have $C_{k}(\Psi) \geq C_{k}(\Phi), k=1, \cdots, d$, we are not sure $|\Psi\rangle \rightarrow|\Phi\rangle$. Here we give an example

$$
\begin{array}{r}
\left|\Phi^{\prime}\right\rangle=\sqrt{0.5}|00\rangle+\sqrt{0.4}|11\rangle+\sqrt{0.1}|22\rangle, \\
\left|\Psi^{\prime}\right\rangle=\sqrt{0.55}|00\rangle+\sqrt{0.3}|11\rangle+\sqrt{0.15}|22\rangle .
\end{array}
$$

One can find the following relations

$$
\begin{array}{r}
C_{2}\left(\Psi^{\prime}\right)=0.2925>C_{2}\left(\Phi^{\prime}\right)=0.29 \\
C_{3}\left(\Psi^{\prime}\right)=0.02475>C_{3}\left(\Phi^{\prime}\right)=0.020 .
\end{array}
$$

According to Nielsen theorem neither $\left|\Psi^{\prime}\right\rangle \rightarrow\left|\Phi^{\prime}\right\rangle$ nor $\left|\Phi^{\prime}\right\rangle \rightarrow\left|\Psi^{\prime}\right\rangle$. That means the concurrence hierarchy is not complete. In the sense of classification pure bipartite states by LOCC, Nielsen theorem is more powerful. However, our result is mainly to quantify the entanglement by concurrence hierarchy.

\section{SUMMUARY AND DISCUSSIONS}

The drawback of the concurrence hierarchy is that it is not complete though the hierarchy consists of $d-1$ independent invariants. We shoud note that Vidal [19], Jonathan and Plenio [20] and Hardy [22] found a complete set of entanglement measures consists of $d-1$ independent entanglement monotones. In concurrence hierarchy, each level of concurrence involves all parameters of a given pure state. So, we can say that each concurrence in the hierarchy describes the entanglement globally. For example, $C_{2}(\Phi)$ describes all 2 level entanglement in a pure state $|\Phi\rangle$. If two eigenvalues between $\lambda_{\Phi}^{\downarrow}$ and $\lambda_{\Phi}^{\downarrow}$ are different, the concurrences in the hierarchy generally will be different.

In summary, we give the definition of concurrence hierarchy. And we propose to use the concurrence hierarchy as measures of entanglement. All concurrences in the hierarchy are zeros for separable states except the normalizaiton one. The concurrence hierarchy is invariant under local unitary transformations. The concurrence hierarchy cannot increase by using LOCC. A simple and direct formula (15) is obtained for concurrence hierarchy. We also analyze some interesting examples by using concurrence hierarchy.

Our result in this paper is a small step toward completely quantifying the entanglement. And we find some interesting applications of concurrence hierarchy. There are a lot of works need to be done along the direction of this paper. We just consider the case of pure states. To study the concurrence hierarchy for mixed state is difficult presently. Because even the first non-trivial concurrence of a general mixed state in d-dimension has not been obtained. We even do not have a widely accepted operational way to find whether a state is entangled. However, our result has potential applications for mixed states. In particular, we give the definition of concurrence hierarchy (13), it could shed light on how we should formulate them for mixed states. We should note that the definition of concurrence hierarchy (13) is just for pure state. To calculate the concurrence hierarchy for mixed states, we need some formulas like the form of Wootters in 2-dimension (3). Because we can not characterize separability only by the eigenvalues of density matrix and reduced density matrices [32].

As we already mentioned, even in classification of pure states by LOCC, the theorem 2 is weaker than Nielsen theorem though it has interesting applications. But we actually raise an interesting question, both $|\Psi\rangle$ and $|\Phi\rangle$ in (17) and $\left|\Psi^{\prime}\right\rangle$ and $\left|\Phi^{\prime}\right\rangle$ in $(20)$ are incomparable by Nielsen theorem. However, by using concurrence hierarchy, we show case (17) and case (20) are belong to different groups. Then what's the essential differences between the case (17) and the case 20,?

It is also interesting to consider other series of quantities to quantify entanglement, for example, we can use invariants $I_{k}=\operatorname{Tr}\left(\Lambda \Lambda^{\dagger}\right)^{k+1}$ as measures of entanglement. And quantum Rényi entropies defined as $S_{j}=\frac{1}{1-j} \log _{2} \operatorname{Tr}\left(\Lambda \Lambda^{\dagger}\right)^{j}$, see for example [25,33], also provide measures of entanglement. Hopefully, quantum Rényi entropies can constitute a complete set of measures of entanglement. And these measures of entanglement work very well for the examples appeared in this paper, i.e., they can determine whether a pure state can be transformed to another by LOCC. However, a proof of whether quantum Rényi entropies is complete or not is necessary. It's also interesting to study whether we can use concurrence hierarchy to study the mixed states, the result in Ref. [34] may be useful to this problem. Some results about invariants for multipartite states are already available [35], it is worth to study the corresponding concurrence hierarchy for multipartite states. 
Acknowlegements: We would like to thank J.Gruska, M.Hamada, W.Y.Hwang, T.Shimono, X.B.Wang, A.Winter and H.Yura for numerous useful discussions, and stimulating talks in ERATO internal seminars. We also thank M.M.Sinolecka, K.Zyczkowski, M.Kus for kind communications.

[1] W.K.Wootters, Phys.Rev.Lett.80, 2245 (1998).

[2] S.Hill, and W.K.Wootters, Phys.Rev.Lett.78, 5022 (1997).

[3] M.A.Nielsen and I.L.Chuang, Qauntum Computation and Quantum Information (Cambrige University Press, Cambridge, England, 2000).

[4] C.H.Bennett, D.P.DiVincenzo, J.A.Smolin, and W.K.Wootters, Phys.Rev.A54, 3824 (1996).

[5] C.H.Bennett, H.J.Bernstein, S.Popescu, and B.Schumacher, Phys.Rev.A53, 2046 (1996).

[6] B.M.Terhal and K.G.H.Vollbrecht, Phys.Rev.Lett.85, 2625 (2000).

[7] K.G.H.Vollbrecht and R.F.Werner, Phys.Rev.A64, 062307 (2001).

[8] C.Bennett, G.Brassard, C.Crepeau, R.Jozsa, A.Peres and W.K.Wootters, Phys.Rev.Lett. 70, 1895 (1993).

[9] A.Ekert, Phys.Rev.Lett.67, 661 (1991).

[10] A.Peres, Phys.Rev.Lett.77,1413(1996).

[11] M.Horodecki, P.Horodecki and R.Horodecki, Phys.Lett.A223,1(1996).

[12] P.Badziag, P.Deuar, M.Horodecki, P.Horodecki, and R.Horodecki, quant-ph/0107147.

[13] A.Uhlmann, Phys.Rev.A62, 032307 (2000).

[14] P.Rungta, V.Bužek, C.M.Caves, M.Hillery, G.J.Milburn, Phys.Rev.A64, 042315 (2001).

[15] S.Albererio and S.M.Fei, J.Opt.B3, 223(2001).

[16] A.F.Absouraddy, B.E.A.Saleh, A.V.Sergienko, and M.C.Teich, Phys.Rev.A64, 050101(R) (2001).

[17] P.Zanardi, C.Zalka, and L.Faoro, Phys.Rev. A62, 030301(R) (2000).

[18] W.K.Wootters, Quantum Inf.Comp.1, 27 (2001).

[19] G.Vidal, Phys.Rev.Lett.83,1046 (1999).

[20] D.Jonathan, M.B.Plenio, Phys.Rev.Lett.83,1455 (1999).

[21] D.Jonathan, M.B.Plenio, Phys.Rev.Lett.83,3566 (1999).

[22] L.Hardy, Phys.Rev.A60,1912 (1999).

[23] G.Vidal, J.Mod.Opt.47,355 (2000).

[24] F.Verstraete, J.Dehaene, B.De Moor, quant-ph/0105090.

[25] K.Zyczkowski, I.Bengtsson, Annals of Phys. 295, 115-135 (200).

[26] M.M.Sinolecka, K.Zyczkowski, M.Kus, Acta Phys. Pol. B33, 2081 (2002), quant-ph/0110082.

[27] G.Vidal,M.Nielsen, QIC 1, 76 (2001).

[28] M.Kus, K.Zyczkowski, Phys.Rev.A63, 032307 (2001).

[29] N.Linden and S.Popescu, Fortsch. Phys.46, 567 (1998).

[30] M.A.Nielsen, Phys.Rev.Lett.83, 436 (1999).

[31] R.Bhatia, Matrix Analysis (Springer-Verlag, New York, 1997).

[32] M.A.Nielsen, and J.Kempe, Phys.Rev.Lett.86, 5184 (2001).

[33] P.Hayden, and A.Winter, On the communication cost of entanglement transformations, quant-ph/0204092.

[34] C.A.Fuchs and K.Jacobs, Phys.Rev.A63, 062305 (2001).

[35] H.Barnum and N.Linden, Monotones and invariants for multi-particle quantum states, quant-ph/0103155. 